\title{
Actividad microbiológica y biomasa microbiana en suelos cafetaleros de los Andes venezolanos
}

\section{Microbial activity and microbial biomass in coffee soils of the Venezuelan Andes}

\author{
Jorge E. Paolini Gómez ${ }^{1 *}$
}

\footnotetext{
${ }^{1}$ Instituto Venezolano de Investigaciones Científicas (IVIC), Centro de Ecología. Apdo. 21825. 1020-A Caracas, Venezuela.

* Autor responsable (jpaolini@ivic.gob.ve)
}

\section{RESUMEN}

En Venezuela el café (Coffea arabica L.) ocupa un área aproximada de 200000 ha, distribuidos principalmente en las zonas montañosas de Los Andes. Tradicionalmente, el café se cultiva con pocos insumos bajo la sombra de árboles de la familia de las leguminosas y de otros arbustos. En años recientes con la adopción de cultivares de alta productividad, notable precocidad y tamaño reducido se está produciendo café a plena exposición solar con fertilización mineral y uso de pesticidas. Las propiedades microbiológicas del suelo especialmente aquellas relacionadas con el flujo de energía y el reciclaje de nutrientes responden de forma rápida $\mathrm{y}$ sensible a los cambios de las condiciones del suelo mucho antes que por ejemplo el carbono orgánico, y de este modo, suministran una información anticipada sobre las alteraciones de la calidad del mismo. En este estudio se comparó la actividad microbiológica (respiración basal), la biomasa microbiana y los parámetros ecofisiológicos en suelos a nivel superficial $(0-5 \mathrm{~cm})$ de ocho fincas cafetaleras en los municipios de Mérida y Trujillo donde se encuentran tres sistemas de producción: tradicional, convencional y orgánico. Las fincas con agricultura orgánica presentaron los mayores valores de carbono orgánico total, carbono hidrosoluble, respiración basal y biomasa microbiana en comparación con las de manejo tradicional y convencional; con lo cual la producción orgánica es un sistema de manejo más sostenible por la mayor conservación de los recursos naturales y la producción más amigable y armónica con el ambiente. En el caso del manejo convencional, la adición de fertilizantes y pesticidas afecta de forma negativa el bienestar de los microorganismos

Cita recomendada:

Paolini Gómez, J. E. 2017. Actividad microbiológica y biomasa microbiana en suelos cafetaleros de los Andes venezolanos. Terra Latinoamericana 36: 13-22.

DOI: https://doi.org/10.28940/terra.v36i1.257 y los hace más ineficientes en el uso del carbono y la energía.

Palabras claves: indicadores biológicos del suelo, respiración basal, cociente microbiano, coeficiente de eficiencia metabólica.

\section{SUMMARY}

In Venezuela approximately 200000 ha are planted with coffee (Coffea arabica L.) mostly on the steep slopes of the Andes. Traditionally, coffee is cultivated with few or no agrochemical inputs in an agroforestry system, cropped under the shade of tree species of the leguminous family and other bushes. In recent years, with adoption of high yielding, early varieties of short stature, coffee is being produced in full sunlight with addition of mineral fertilizers and pesticides. The soil microbiological properties especially those related with the fluxes of energy and nutrient cycling, respond promptly to changes in soil conditions, often much earlier than organic carbon and, in this way, are early indicators of alterations in soil quality. In this study, we compared the microbiological activity (basal respiration), microbial biomass and derived ecophysiological ratios of topsoils $(0-5 \mathrm{~cm})$ collected from eight farms in the municipalities of Merida and Trujillo that practice traditional (agroforestry or shaded coffee), conventional (sun coffee or intensive monoculture) and organic (agroforestry or shaded coffee) production. The organic agriculture farms showed the highest contents of total organic carbon, water soluble carbon, basal respiration and microbial biomass compared with traditional and conventional farms. This means a more sustainable coffee system

Recibido: marzo de 2017. Aceptado: noviembre de 2017. Publicado en Terra Latinoamericana 36: 13-22. 
with better conservation of natural resources and environment-friendly production. On conventional farms, added fertilizers and pesticides negatively affect microorganism performance and are more inefficient in terms of carbon and energy use.

Index words: soil biological indicators, basal respiration, microbial quotient, microbial efficient quotient.

\section{INTRODUCCIÓN}

El café constituyó durante los siglos XVIII, XIX y hasta principios del siglo XX uno de los productos de exportación agrícola más importantes de la economía venezolana; desde la época colonial se estableció en las regiones montañosas del país. Los cambios económicos experimentados en el país, fruto de la explotación petrolera a principios del siglo XX, causaron una declinación de la producción agrícola en general. En Venezuela, al igual que en muchos países de Centro y Sur de América, el café tradicionalmente se cultiva bajo sombra de árboles del género Inga, Cordia, Cedrela y Erythrina aunque también de árboles frutales como aguacate (Persea americana), cítricos (Citrus sinensis) y bananos (Musa paradisiaca); conocido como sistema cafetalero multiestrato o policultivo tradicional, el cual mantiene la biodiversidad funcional y estructural del ecosistema (Escalante, 1985; Moguel y Toledo, 1999; Beer et al., 1998; Perfecto y Armbrech, 2003). Estos árboles proveen de sombra al cultivo y mejora la calidad del café, reciclan nutrientes de las capas profundas del suelo, protegen al arbusto del café del viento, la lluvia y del exceso de sol, ayudan a regular la variabilidad climática, estabilizan el suelo al reducir la erosión y la densidad aparente, incrementan la infiltración del agua y proveen de nitrógeno al cultivo a través de la fijación biológica de este (Beer, 1987; Beer et al., 1998; DaMatta, 2004; MamaniPati et al., 2012; Rapidel et al., 2015). La región andina venezolana es, por excelencia, productora de café y sus suelos se caracterizan por poseer $\mathrm{pH}$ ácidos, texturas arcillo-limosa, con poco o incipiente desarrollo. A partir de 1975 en Venezuela se comenzó a reemplazar los cafetales bajo sombra con variedades de café de alta productividad, resistentes a plagas y enfermedades como Bourbon, Caturra, Catuaí y Mundo Novo; las cuales además crecen a plena exposición solar con densidades elevadas de plantas y altos requerimientos de agroquímicos como fertilizantes y pesticidas (Henao-Jaramillo, 1996) lo que conduce irremediablemente a la degradación del suelo, contaminación ambiental y problemas de salud humana. Actualmente algunos agricultores están cultivando café de forma orgánica, el interés radica en producir granos de una máxima calidad nutricional, manteniendo la calidad del suelo y respetando el ambiente (Muschler, 2001; Bosselman et al., 2009; Haggar et al., 2011) y de ese modo pueden evadir las fluctuaciones del mercado y la especulación de los precios. A nivel mundial en el año 2014 se cultivaron 9900000 ha con café, de los cuales 7.7\% (763000 ha) corresponden a café orgánico y dicha superficie continua en ascenso; en la década comprendida entre los años 2004-2014 la misma se cuadruplicó (Willer y Lernoud, 2016).

En la mayoría de los suelos, los microorganismos dominan el componente biológico de los mismos y responden rápidamente a los cambios del ambiente. Ellos son esenciales para realizar las múltiples funciones del suelo, participan en casi todas las reacciones metabólicas conocidas y constituyen las fuerzas motrices del suministro de energía y nutrientes. La degradación de la materia orgánica del suelo (MOS) es un proceso inherente a todos los microorganismos heterotróficos y generalmente su tasa o velocidad de descomposición indica el nivel de la actividad microbiana de un suelo. Entre las técnicas más usadas para medir la descomposición o mineralización de la MOS está la medición del consumo de $\mathrm{O}_{2}$ o el desprendimiento de $\mathrm{CO}_{2}$ y su determinación en condiciones controladas de laboratorio, se conoce con el nombre de actividad microbiana o respiración basal y es el mejor indicador de la actividad metabólica global de las comunidades microbianas del suelo (Nannipieri et al., 1990; Alef, 1995; Pell et al., 2006). Por otro lado, hay que considerar que la respiración de los microorganismos es el eslabón fundamental que cierra el ciclo del carbono en los ecosistemas terrestres, retornándolo a la atmósfera en forma de $\mathrm{CO}_{2}$.

Otro parámetro microbiológico que se mide en conjunto con la respiración basal es la biomasa microbiana $\left(\mathrm{C}_{\text {mic }}\right)$, que cuantifica la cantidad global de microorganismos presentes en un suelo, en otras palabras, el tamaño de la comunidad microbiana presente en el suelo, se considera el componente vivo de la MOS y representa en promedio del 1 al $4 \%$ del carbono orgánico total (COT) del suelo (Jenkinson y Ladd, 1981). A pesar de ser una pequeña fracción es más sensible y responde más rápidamente que 
el carbono orgánico total y el nitrógeno total para inferir alteraciones de la MOS causada por las prácticas de manejo.

El objetivo del presente estudio fue evaluar los efectos de tres sistemas de producción de café: orgánico, tradicional y convencional sobre el carbono orgánico total, el carbono hidrosoluble, la respiración basal y la biomasa microbiana en suelos a nivel superficial $(0-5 \mathrm{~cm})$. Adicionalmente se calcularon las diferentes constantes ecofisiológicas, las cuales integran componentes abióticos y bióticos y definen cada sistema, como son: el cociente microbiano (qMic), el cociente metabólico $\left(\mathrm{qCO}_{2}\right)$ y el cociente de eficiencia metabólica $\left(\mathrm{qCO}_{2} \mathrm{C}_{\text {org }}{ }^{-1}\right)$.

\section{MATERIALES Y MÉTODOS}

\section{Sitio de Estudio}

En el estado de Mérida se seleccionaron dos sistemas de manejo, uno en las cercanías de la población de La Azulita (Municipio Andrés Bello; Precipitación: $1495 \mathrm{~mm}$ ) en tres fincas certificadas bajo manejo orgánico (sistema agroforestal multiestrato, con café de 6-8 años de edad y adición de 250-500 g planta $^{-1}$ de estiércoles animales, vermicomposts, residuos del beneficio del café y abonos verdes: Crotalaria juncea, Cannavalia ensiformes, Vigna unguiculata, Arachis pintoi entre otros) pertenecientes a la Asociación de Producción Orgánica Quebrada Azul y una finca en Santa Cruz de Mora (Municipio Pinto Salinas; Precipitación: 1100-1350 mm; temperatura: 8-24 ${ }^{\circ} \mathrm{C}$ ), plantación con parcelas de 1 a 5 años de edad, bajo manejo convencional (uso de fertilizantes: $500-800 \mathrm{~kg} \mathrm{ha}^{-1}$ año $0^{-1}$ de formula completa $15-15-15$ y agroquímicos) con café a plena exposición solar. En el estado Trujillo se seleccionaron tres fincas en la microcuenca del río Monaicito (Municipio Pampán; Precipitación: $1022 \mathrm{~mm}$; temperatura: $16-25^{\circ} \mathrm{C}$ ) bajo manejo tradicional (sistema agroforestal multiestrato o café bajo sombra de árboles, con pequeñas adiciones de fertilizantes sintéticos $\left(20-50 \mathrm{~g}\right.$ planta $\left.{ }^{-1}\right)$, control manual de malezas y edad promedio de la plantación mayor de 25 años).

\section{Muestreo}

En cada sistema de manejo se recolectaron de nueve a doce muestras compuestas a nivel superficial $(0-5 \mathrm{~cm})$ bajo el dosel de los arbustos de café.
Se escogió dicha profundidad de muestreo puesto que los mayores contenidos de materia orgánica y actividad biológica se exhiben en los primeros centímetros del suelo (Van Gestel et al., 1992; Böhme et al., 2005; Madejón et al., 2007; Payán et al., 2009). Las muestras fueron trasladadas al laboratorio del IVIC en bolsas plásticas, cernidas por un tamiz de $2 \mathrm{~mm}$ y una alícuota de $250 \mathrm{~g}$ fue preservada en refrigeración a $4{ }^{\circ} \mathrm{C}$ durante cuatro semanas hasta la evaluación de las propiedades biológicas y el resto de la muestra fue secada al aire a temperatura del laboratorio para los análisis de carbono orgánico total y carbono hidrosoluble.

\section{Determinaciones}

El carbono orgánico total $\left(\mathrm{C}_{\text {org }}\right)$ se determinó mediante la oxidación húmeda de 0.1 a 0.2 g suelo seco con $4 \mathrm{~mL}$ ácido sulfúrico concentrado y $2 \mathrm{~mL}$ dicromato de potasio $2 \mathrm{~N}$ y la cantidad de carbono orgánico oxidado por el $\mathrm{Cr}(\mathrm{VI})$ se midió espectrofotométricamente a $600 \mathrm{~nm}$, donde el ión $\mathrm{Cr}$ (III) formado durante la reacción presenta su máxima absorbancia. Se comparó el color con las lecturas de una curva de calibración preparada con glucosa, sometidas al mismo tratamiento que las muestras (Sims y Haby, 1971).

Para extraer el carbono hidrosoluble (CHS) se agitó la muestra de suelo seca en tubos de centrifuga durante $1 \mathrm{~h}$, en una relación suelo/agua de 1:4. Después de centrifugar a $3500 \mathrm{rpm}$ durante $15 \mathrm{~min}$; el sobrenadante se filtró a través de filtros Millipore de $0.45 \mathrm{~m} \mu$. El carbono orgánico se midió por el método espectrofotométrico descrito en el párrafo anterior para el $\mathrm{C}_{\mathrm{org}}$.

La respiración basal $(\mathrm{RB})$ se midió por el método de trampa de álcali, en donde $20 \mathrm{~g}$ de suelo se colocaron en un frasco de vidrio hermético de $500 \mathrm{~mL}$ de capacidad, se ajustó con agua destilada al $50-60 \%$ de su capacidad de retención de humedad y se incubó por un período de siete días a temperatura de $22 \pm 2{ }^{\circ} \mathrm{C}$ en oscuridad (Alef, 1995; Pell et al., 2006). El $\mathrm{CO}_{2}$ liberado se atrapó en un vial que contenía $10 \mathrm{~mL}$ de $0.2 \mathrm{M} \mathrm{NaOH}$; al final del período de incubación el vial se trasvasó cuantitativamente a un matraz Erlenmeyer, se añadió $2 \mathrm{~mL} 0.5 \mathrm{M} \mathrm{BaCl}_{2}$ para precipitar el $\mathrm{CO}_{2}$ y el exceso de hidróxido se tituló con $0.2 \mathrm{~N} \mathrm{HCl}$ patrón usando fenolftaleína como indicador. La determinación se realizó por triplicado. La cantidad de $\mathrm{CO}_{2}$ liberado se calculó mediante la fórmula: 
$\mathrm{mg} \mathrm{C}-\mathrm{CO}_{2}=\left(\mathrm{V}_{\mathrm{B}}-\mathrm{V}_{\mathrm{M}}\right) \times 6 \times \mathrm{N}_{\mathrm{HCl}}$

donde:

$\mathrm{V}_{\mathrm{B}}=$ cantidad de ácido clorhídrico gastado para valorar el blanco (frasco sin suelo),

$\mathrm{V}_{\mathrm{M}}=$ cantidad de ácido clorhídrico gastado para valorar la muestra del suelo,

$6=$ peso equivalente del carbono,

$\mathrm{N}_{\mathrm{HCl}}=$ normalidad del ácido clorhídrico.

Los resultados se expresan en $\mathrm{mg} \mathrm{C}-\mathrm{CO}_{2} \mathrm{~kg}^{-1}$ suelo seco día ${ }^{-1}$.

Labiomasa microbianase determinó por el método de respiración inducida por sustrato (Anderson y Domsch, 1978; Beck et al., 1995; Höper, 2006) y se usó el mismo suelo proveniente de la respiración basal al cual se le añadió $1 \mathrm{~mL}$ glucosa $8 \%$ y el $\mathrm{CO}_{2}$ liberado en seis horas se estima por el mismo método (trampa de álcali) pero la concentración de $\mathrm{NaOH}$ fue de $0.05 \mathrm{~N}$ y el $\mathrm{HCl} 0.05 \mathrm{~N}$.

Se usó el factor de conversión propuesto por Beck et al. (1995) para el cálculo de la biomasa microbiana:

$1 \mathrm{mgC}-\mathrm{CO}_{2} 100 \mathrm{~g}^{-1} \mathrm{ssh}=20.6 \mathrm{mgC}_{\text {mic }} 100 \mathrm{~g}^{-1}$ suelo seco.

Los índices ecofisiológicos fueron calculados como sigue:

$\mathrm{qMic}=\mathrm{C}_{\text {mic }} \mathrm{C}_{\text {org }}^{-1}=\left[\left(\mathrm{mg} \mathrm{C}_{\text {mic }} \mathrm{kg}^{-1}\right)\left(\mathrm{mgC}_{\text {org }} \mathrm{kg}^{-1}\right)^{-1}\right] \times 100$ (Anderson y Domsch, 1989),

$\mathrm{qCO}_{2}=\mathrm{RBC}_{\text {mic }}{ }^{-1}=\mathrm{mgC}-\mathrm{CO}_{2 \text { basal }} \times \mathrm{g}^{-1} \mathrm{C}_{\text {mic }} \times \mathrm{h}^{-1}$ (Anderson y Domsch, 1990; Dilly y Munch, 1998), $\mathrm{qCO}_{2} \mathrm{C}_{\text {org }}{ }^{-1}=\left(\mathrm{mgC}^{-} \mathrm{CO}_{2 \text { basal }} \times \mathrm{g}^{-1} \mathrm{C}_{\text {mic }} \times \mathrm{h}^{-1}\right)\left(\mathrm{g} \mathrm{C}_{\text {org }} \mathrm{g}^{-1}\right)^{-1}$ (Dilly, 2005).

Estos índices se consideran como indicadores "potenciales" de las propiedades biológicas del suelo y sus procesos, ya que ellos se obtienen de suelos analizados en el laboratorio en condiciones estándares (tamizado, temperatura y humedad controlada) las cuales no necesariamente reflejan las condiciones in situ (Nannipieri et al., 1990; Moscatelli et al., 2005).

Los datos fueron analizados con el paquete estadístico SPSS Versión 15.0 mediante un análisis de varianza (ANDEVA) de un factor (sistema de manejo) previa comprobación de los supuestos de normalidad con el test Shapiro-Wilk y homosedasticidad con el test de Levene; en caso de la violación de este supuesto los datos fueron transformados a logaritmos para su análisis. En la comparación de medias se aplicó la prueba de mínima diferencia significativa (MDS) a un nivel de $0.05 \%$. Adicionalmente se utilizó la correlación de Pearson para establecer el grado y tipo de asociación entre cada variable.

\section{RESULTADOS Y DISCUSIÓN}

\section{Carbono Orgánico $\left(\mathrm{C}_{\text {org }}\right)$}

El contenido de $\mathrm{C}_{\text {org }}$ resultó ser estadísticamente mayor en los suelos bajo tratamiento orgánico con respecto al resto de los tratamientos (Cuadro 1), lo que se corresponde con las continuas adiciones de estiércoles animales, vermicomposts, residuos del beneficio del café y abonos verdes y la caída de la hojarasca de café y árboles de sombra. Los suelos con sistemas de manejo tradicional y convencional mostraron valores sin diferencias significativas entre ellos (Cuadro 1); aunque en el caso del sistema tradicional hay un aporte de materia orgánica por la hojarasca de café y los árboles de sombra, que en algunos sistemas agroforestales cafetaleros

Cuadro 1. Valores medios ( \pm desviación estándar) de carbono orgánico, carbono hidrosoluble, respiración basal, biomasa microbiana y de los parámetros ecofisiológicos de los cafetaleros estudiados.

\begin{tabular}{|c|c|c|c|c|}
\hline \multirow{2}{*}{ Parámetro } & \multirow{2}{*}{ Unidades } & \multicolumn{3}{|c|}{ Sistema de producción } \\
\hline & & Orgánico (n = 9) & Tradicional $(\mathrm{n}=12)$ & Convencional $(n=9)$ \\
\hline $\mathrm{C}_{\text {org }}$ & $\mathrm{g} \mathrm{C} \mathrm{kg}^{-1}$ & $66.6 \pm 8.6 \mathrm{a} \dagger$ & $35.0 \pm 11.7 \mathrm{~b}$ & $29.0 \pm 3.7 b$ \\
\hline CHS & $\mathrm{mg} \mathrm{C} \mathrm{kg-1}$ & $872 \pm 134 \mathrm{a}$ & $351 \pm 111 b$ & $177 \pm 52 \mathrm{c}$ \\
\hline $\mathrm{RB}$ & $\mathrm{mg} \mathrm{C}-\mathrm{CO}_{2} \mathrm{~kg}^{-1} \mathrm{~d}^{-1}$ & $76.2 \pm 20.4 \mathrm{a}$ & $55.1 \pm 10.2 b$ & $51.2 \pm 8.3 b$ \\
\hline $\mathrm{C}_{\text {mic }}$ & $\mathrm{mg} \mathrm{C}_{\mathrm{mic}} \mathrm{kg}^{-1}$ & $472 \pm 163$ a & $463 \pm 132 \mathrm{a}$ & $246 \pm 79 b$ \\
\hline $\mathrm{q}_{\text {Mic }}$ & $\%$ & $0.71 \pm 0.22 b$ & $1.35 \pm 0.21 \mathrm{a}$ & $0.85 \pm 0.23 b$ \\
\hline $\mathrm{qCO}_{2}$ & $\mathrm{mg} \mathrm{C}-\mathrm{CO}_{2} \mathrm{~g}^{-1} \mathrm{Cmic} \mathrm{h}^{-1}$ & $7.9 \pm 4.2 \mathrm{ab}$ & $5.3 \pm 1.4 \mathrm{~b}$ & $9.6 \pm 3.5 \mathrm{a}$ \\
\hline $\mathrm{qCO}_{2} / \mathrm{C}_{\text {org }}$ & $\left(\mathrm{mg} \mathrm{C}-\mathrm{CO}_{2} \mathrm{~g}^{-1} \mathrm{C}_{\text {mic }} \mathrm{h}\right)\left(\mathrm{g} \mathrm{C}_{\text {org }} \mathrm{g}^{-1}\right)^{-1}$ & $123 \pm 69 b$ & $180 \pm 103 b$ & $339 \pm 144 \mathrm{a}$ \\
\hline
\end{tabular}

$\overline{\mathrm{C}_{\text {org }}}=$ carbono orgánico total; $\mathrm{CHS}=$ carbono hidrosoluble; $\mathrm{RB}=$ respiración basal; $\mathrm{C}_{\text {mic }}=$ carbono microbiano; $\mathrm{qMic}=$ cociente microbiano; $\mathrm{qCO}_{2}=$ cociente metabólico; $\mathrm{qCO}_{2} / \mathrm{C}_{\text {org }}=$ cociente de eficiencia metabólica. ${ }^{\dagger}$ Letras distintas en la misma fila indican diferencias significativas entre los sistemas (ANDEVA de un factor $P \leq 0.05$ y comparación de medias con la prueba de MDS). 
venezolanos está en el orden de 11.2 a $17.4 \mathrm{Mg}_{\text {ha }}{ }^{-1}$ año ${ }^{-1}$ (Aranguren et al., 1982; Arellano et al., 2004) comparado con $5.7 \mathrm{Mg} \mathrm{ha}^{-1}$ año $^{-1}$ en café a plena exposición solar (Quintero y Ataroff, 1998). Varios autores han confirmado mayores contenidos de $\mathrm{C}_{\text {org }}$ en sistemas bajo manejo orgánico al ser contrastados con sistemas convencionales (Berner et al., 2008; Canali et al., 2009; Lagomarsino et al., 2009; Leite et al., 2010; Melero et al., 2006; Payán et al., 2009; Haggar et al., 2011; Santos et al., 2012). Algunos autores como Kashuk et al. (2010) indican que, en ecosistemas agrícolas, el carbono orgánico del suelo es la restricción o limitación fundamental a la biomasa microbiana.

\section{Carbono Hidrosoluble (CHS)}

El carbono hidrosoluble estimula el desarrollo y la actividad de las poblaciones microbianas presentes en el suelo y se considera que esta fracción está constituida por compuestos de bajo peso molecular y altamente lábiles como azúcares, aminoácidos y lípidos. Las fincas bajo manejo orgánico presentaron los mayores valores de este parámetro seguido de las fincas bajo manejo tradicional (Cuadro 1); asociado a un mayor aporte de materia orgánica por la adición de enmiendas orgánicas y la hojarasca de los árboles de sombra y café, y el menor valor en la finca convencional, donde solo hay aporte de hojarasca de café. Los valores de este estudio se asemejan a los reportados por Pabst et al. (2013) para cafetales y jardines domésticos ubicados en las laderas del Monte Kilimanjaro entre Kenia y Tanzania de 875 $\pm 160 \mathrm{mg} \mathrm{kg}^{-1}$ y $430 \pm 66 \mathrm{mg} \mathrm{kg}^{-1}$, respectivamente.

\section{Actividad Microbiana o Respiración Basal (RB)}

La respiración basal varió entre 35.8 y $110.7 \mathrm{mg} \mathrm{C}-\mathrm{CO}_{2} \mathrm{~kg}^{-1} \mathrm{~d}^{-1}$, siendo mayor en las fincas bajo manejo orgánico en comparación con los otros dos manejos (Cuadro 1); lo cual indica una mayor actividad microbiana del suelo en este sistema debido a la adición permanente y continua de fuentes exógenas de materia orgánica lábil al suelo y la subsecuente estimulación de los microorganismos heterotróficos (Araújo et al., 2008; Bettiol et al., 2002; Saffigna et al., 1989; Okur et al., 2015). Los altos valores de la respiración basal en los suelos bajo manejo orgánico están relacionados con el mayor contenido de carbono orgánico, carbono hidrosoluble y biomasa microbiana de estos suelos.
Los valores de este estudio son similares a los reportados por Almeida et al. (2007) en Minas de Gerais (Brasil) para sistemas agroforestales de café $\left(37.5 \mathrm{mg} \mathrm{C}-\mathrm{CO}_{2} \mathrm{~kg}^{-1} \mathrm{~d}^{-1}\right)$ y café a plena exposición solar (43.7 mg C-CO $\mathrm{kg}^{-1} \mathrm{~d}^{-1}$ ) y superan los valores reportados por Glaesser et al. (2010) en Mato Grosso (Brasil) de 4.9 a $21.7 \mathrm{mg} \mathrm{C}-\mathrm{CO}_{2}$ $\mathrm{kg}^{-1} \mathrm{~d}^{-1}$ para café orgánico en oxisoles arenosos, por Nunes et al. (2009) en el estado de Minas de Gerais (Brasil) para monocultivo de café durante 16 y 22 años de 8.2 a 24.5 mg C-CO $\mathrm{kg}^{-1} \mathrm{~d}^{-1}$ y Severino y Oliveira (1999) en el estado de Ceará (Brasil) de 7.4 y $12.0 \mathrm{mg} \mathrm{C}-\mathrm{CO}_{2} \mathrm{~kg}^{-1} \mathrm{~d}^{-1}$ en cafetales longevos bajo sombra de Inga spp. y bananos. Recientemente Velmourougane (2016) en la India encontró diferencias estadísticamente significativas al comparar cafetales con manejo orgánico (valor promedio de $53.3 \mathrm{mg} \mathrm{C}-\mathrm{CO}_{2} \mathrm{~kg}^{-1} \mathrm{~d}^{-1}$ ) con cafetales bajo manejo convencional (valor promedio $\left.45.1 \mathrm{mg} \mathrm{C}-\mathrm{CO}_{2} \mathrm{~kg}^{-1} \mathrm{~d}^{-1}\right)$.

\section{Biomasa Microbiana $\left(\mathrm{C}_{\text {mic }}\right)$}

La biomasa microbiana fue similar tanto en el manejo orgánico como en el tradicional no detectándose diferencias significativas entre ambos tipos de sistema; no así cuando se compararon con la finca bajo manejo convencional donde la biomasa microbiana resultó ser casi la mitad repecto al manejo orgánico y tradicional (Cuadro 1). La biomasa microbiana se considera que es una fracción lábil de la materia orgánica del suelo y es un reservorio importante de nutrientes para los vegetales, como $\mathrm{N}$ y $\mathrm{P}$ (Marumoto et al., 1982), así mismo tiene importantes funciones en el suelo, entre las cuales podemos nombrar el reciclaje de nutrientes y la degradación de contaminantes como pesticidas, residuos sólidos urbanos e industriales. La biomasa microbiana al ser la parte viva de la materia orgánica del suelo actúa como un indicador ecológico importante y responde tempranamente a cambios en las condiciones del suelo, antes de que pueda ser detectado en el carbono orgánico del suelo (Anderson y Domsch, 1989; Marinari et al., 2006). El mayor contenido de biomasa microbiana en las parcelas bajo manejo orgánico y tradicional está asociado a una mayor incorporación de materia orgánica y nutrientes vía hojarasca de café y árboles de sombra; a pesar de las diferencias en condiciones climáticas, tipos de suelo y cultivos, numerosos estudios sobre agricultura orgánica confirman nuestros hallazgos 
(Gunapala y Scow, 1998; Mäder et al., 2002; Marinari et al., 2006; Melero et al., 2006; Tu et al., 2006; Araújo et al., 2008; Lagomarsino et al., 2009; Leite et al., 2010; Joergensen et al., 2010; Santos et al., 2012). Los resultados de este estudio se asemejan a los reportados por Theodoro et al. (2003) en sistemas cafetaleros del estado de Minas de Gerais (Brasil) donde el promedio para el sistema orgánico fue de $433 \mathrm{mg} \mathrm{C}_{\text {mic }} \mathrm{kg}^{-1}$ y para el sistema convencional de $372 \mathrm{mg} \mathrm{C}_{\text {mic }} \mathrm{kg}^{-1}$ aunque estos autores no detectaron diferencias significativas entre ambos sistemas y épocas de muestreo (lluvia-seca); por Balota y Chaves (2011) de 102-427 $\mathrm{mg} \mathrm{C}_{\text {mic }} \mathrm{kg}^{-1}$ en el estado de Paraná (Brasil) medidos en suelos superficiales bajo el dosel del café y entre las calles sembrados con diferentes leguminosas y superan a los referidos por Glaeser et al. (2010) para sistemas cafetaleros orgánicos creciendo sobre oxisoles arenosos del estado de Mato Grosso del Sur (Brasil) de 87 a $212 \mathrm{mg} \mathrm{C}_{\text {mic }} \mathrm{kg}^{-1}$ y Partelli et al. (2012) para sistemas de producción convencional y orgánico de café conilon (Coffea canephora) en el estado de Espirita Santo (Brasil) de 73 a $189 \mathrm{mg} \mathrm{C}_{\text {mic }} \mathrm{kg}^{-1}$ respectivamente, pero son inferiores a los reportados por Sánchez et al. (2006) en Costa Rica de $583 \mathrm{mg} \mathrm{C}_{\text {mic }} \mathrm{kg}^{-1}$ para un sistema agroforestal convencional y de $913 \mathrm{mg} \mathrm{C}_{\text {mic }} \mathrm{kg}^{-1}$ para un sistema agroforestal orgánico.

\section{Cociente Microbiano (qMic)}

La relación $\mathrm{C}_{\text {mic }} \mathrm{C}_{\text {org }}^{-1}$, denominada cociente microbiano (qMic), es un indicador de la disponibilidad de carbono de los microorganismos, entradas de materia orgánica al suelo, eficiencia en la conversión del carbono a biomasa microbiana y de la estabilización del carbono por la fracción mineral del suelo (Anderson, 1994; Anderson y Domsch, 1989; Sparling, 1992). Este cociente resultó ser mayor en el manejo tradicional y similar entre el sistema orgánico y convencional (Cuadro 1); los valores obtenidos en el presente trabajo son relativamente bajos en comparación con el valor umbral de 2 y $4.4 \%$ propuesto para suelos agrícolas y forestales de zonas templadas con $\mathrm{pH}$ neutrales (Anderson, 2003); aunque en la literatura se han reportado para suelos cafetaleros valores similares a los de este estudio por Almeida et al. (2007); Balota y Chaves (2011); Glaesser et al. (2010); Partelli et al. (2012); Pimentel et al. (2006) y Souza et al. (2012) en cafetales brasileños y por Dariah et al. (2007) en Indonesia; lo cual nos podría estar indicando que además del tenor de materia orgánica y atributos de la misma como la calidad química, el estatus nutricional, la textura, la mineralogía y el manejo del suelo influyen sobre el mencionado parámetro ecofisiológico (Sparling, 1992).

\section{Cociente Metabólico $\left(\mathrm{q} \mathrm{CO}_{2}\right)$}

El cociente metabólico $\left(\mathrm{qCO}_{2}\right)$ es quizás el índice metabólico más usado cuando se quiere describir la eficiencia de los microorganismos en el uso de la energía (mantenimiento y crecimiento) con el concomitante aumento de la biodiversidad en la sucesión de ecosistemas hacia la madurez y se aplica cuando se quiere comparar el impacto que tienen cambios estacionales, sistemas de manejo, adición de metales, agroquímicos y xenobióticos sobre los microorganismos del suelo. Este índice varía con la composición y el estado fisiológico de la comunidad microbiana, la disponibilidad de sustratos y varios factores abióticos como $\mathrm{pH}$, textura, temperatura y carbono orgánico(Anderson, 1994). Bajo condiciones desfavorables, los microorganismos requieren de más energía para mantener la biomasa y por lo tanto aumenta el $\mathrm{qCO}_{2}$ y el carbono se pierde como $\mathrm{CO}_{2}$ (Anderson, 1994; Insam y Domsch, 1988). De acuerdo con este índice, en el sistema convencional los microorganismos estarían sujetos a un mayor estrés no así en el sistema tradicional y orgánico. De hecho, en la finca bajo manejo convencional, donde se muestrearon lotes con café recién establecido, se presentaron los mayores cocientes metabólicos (8.5-15 mg C-CO $\mathrm{g}^{-1} \mathrm{C}_{\text {mic }} \mathrm{h}^{-1}$ ); esto corrobora los resultados previamente reportados por Anderson y Domsch (1990) para suelos agrícolas donde las parcelas más jóvenes mostraron los valores más altos de $\mathrm{qCO}_{2}$. En las fincas bajo manejo tradicional, los cafetales son de mayor edad con lo cual podemos considerar que son ecosistemas maduros y estables y presentaron los menores valores de $\mathrm{qCO}_{2}$, en concordancia con los postulados de Odum (1969). Anderson (2003) indica que este índice debería de variar entre 0.5 y $2 \mathrm{mg} \mathrm{C}-\mathrm{CO}_{2} \mathrm{~g}^{-1} \mathrm{C}_{\text {mic }} \mathrm{h}^{-1}$ para suelos de $\mathrm{pH}$ neutro y valores por encima de $2 \mathrm{mg} \mathrm{C}-\mathrm{CO}_{2} \mathrm{~g}^{-1} \mathrm{C}_{\text {mic }} \mathrm{h}^{-1}$ podrían ser considerados como críticos. Otros autores como Leita et al. (1999) y Marinari et al. (2006) indican valores comprendidos 
entre 0.2 y $4.8 \mathrm{mg} \mathrm{C}-\mathrm{CO}_{2} \mathrm{~g}^{-1} \mathrm{C}_{\text {mic }} \mathrm{h}^{-1}$ para suelos agrícolas de las zonas templadas.

Este cociente ha sido criticado por varios autores como Wardle y Ghanni (1995) los cuales demostraron que dicho parámetro no distingue entre estrés y perturbación a nivel del ecosistema. Cheng et al. (1996); Dilly (2005); Dilly y Munch (1996); Dinesh et al. (2003) y Franca et al. (2007) indicaron que cuando la materia orgánica del suelo contiene elevadas cantidades compuestos fácilmente disponibles y degradables este cociente se incrementa. Esto podría explicar el incremento del $\mathrm{qCO}_{2}$ en las fincas bajo manejo orgánico en comparación con las fincas bajo manejo tradicional; ya que adicionalmente a las entradas de $\mathrm{C}$ por la hojarasca de los árboles de sombra y café hay un aporte extra por la adición de vermicompostas, abonos verdes y estiércol animal.

Tanto el cociente microbiano como el metabólico fueron incapaces de detectar diferencias entre el sistema de manejo orgánico y convencional como lo ha sido en otros trabajos (Mäder et al., 2002; Marinari et al., 2006; Araújo et al., 2008).

\section{Cociente de Eficiencia Metabólica $\left(\mathrm{qCO}_{2} \mathrm{C}_{\mathrm{org}^{-1}}\right)$}

Dilly et al. (2001) y Dilly (2005) propusieron un nuevo índice ecofisiológico llamado cociente de eficiencia metabólica, donde el cociente metabólico es normalizado respecto al contenido de carbono orgánico; dicho parámetro integra la respiración basal, la biomasa microbiana y el contenido de carbono orgánico en un solo valor. Los microorganismos son más eficientes desde el punto de vista energético si este cociente es menor, y se incrementa cuando la respiración es mayor, la biomasa microbiana disminuye y el sustrato es menos rico y de menor calidad en carbono orgánico (Dilly, 2005). La relación $\mathrm{qCO}_{2} \mathrm{C}_{\text {org }}^{-1}$ fue más baja en el sistema orgánico atribuible a una mayor diversidad y mayor eficiencia de la microbiota del suelo (Mäder et al., 2002; Velmourougane, 2016) aunque no se detectó diferencias significativas con el sistema tradicional, pero si con el sistema convencional; con lo cual podemos decir que en este último sistema la comunidad microbiana es menos eficiente. En la literatura no se encuentran muchos trabajos que usen este índice, sin embargo, en el norte de Alemania se compararon diversos usos de la tierra (suelos agrícolas, praderas y bosques) encontrándose valores comprendidos entre 33 y $274 \mathrm{mg} \mathrm{C}-\mathrm{CO}_{2} \mathrm{~g}^{-1} \mathrm{C}_{\text {mic }} \mathrm{h}^{-1}\left(\mathrm{gC}_{\text {org }} \mathrm{g}^{-1}\right)^{-1}$ y los autores enfatizan que las comunidades más ineficientes en el uso del carbono eran aquellas en suelos bajo monocultivo de maíz y las más eficientes en praderas húmedas (Dilly et al., 2001). Los valores de este estudio están dentro del intervalo anteriormente reportado.

Todas las variables microbiológicas excepto el $\mathrm{qCO}_{2}$ correlacionaron significativamente con el carbono orgánico e hidrosoluble (Cuadro 2). Otros autores como Jenkinson y Ladd (1981); Anderson y Domsch (1989); Sparling (1992); Leita et al. (1999); Balota y Chaves (2011); Melero et al. (2006) también

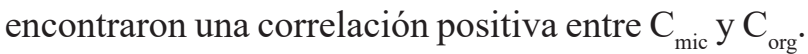
El mayor coeficiente de correlación se presentó entre el carbono orgánico total y el carbono hidrosoluble $(\mathrm{r}=0.950)$ (Cuadro 2).

Cuadro 2. Coeficientes de correlación lineal entre las variables estudiadas $(n=30)$.

\begin{tabular}{llllllll}
\hline & $\mathrm{C}_{\text {org }}$ & $\mathrm{CHS}$ & $\mathrm{RB}$ & $\mathrm{C}_{\text {mic }}$ & $\mathrm{qMic}$ & $\mathrm{qCO}_{2}$ & $\mathrm{qCO}_{2} \mathrm{C}_{\text {org }}{ }^{-1}$ \\
\hline $\mathrm{C}_{\text {org }}$ & 1 & & & & & & \\
$\mathrm{CHS}$ & $0.950^{* * *}$ & 1 & & & & & \\
$\mathrm{RB}$ & $0.656^{* * *}$ & $0.566^{* * *}$ & 1 & & & & \\
$\mathrm{C}_{\text {mic }}$ & $0.582^{* * *}$ & $0.590^{* * *}$ & 0.08 & 1 & & & \\
$\mathrm{qMic}$ & $-0.449^{* *}$ & $-0.331^{* *}$ & $-0.543^{* *}$ & $0.389^{*}$ & 1 & & \\
$\mathrm{qCO}_{2}$ & -0.094 & -0.166 & $0.445^{*}$ & $-0.773^{* * *}$ & $-0.725^{* * *}$ & 1 & \\
$\mathrm{qCO}_{2} \mathrm{C}_{\text {org }}{ }^{-1}$ & $-0.667 * * *$ & $-0.661^{* * *}$ & -0.197 & $-0.865^{* * *}$ & -0.207 & $0.719^{* * *}$ & 1 \\
\hline
\end{tabular}

$\overline{\mathrm{C}_{\text {org }}}=$ carbono orgánico total; $\mathrm{CHS}=$ carbono hidrosoluble; $\mathrm{RB}=$ respiración basal; $\mathrm{C}_{\text {mic }}=$ carbono microbiano; $\mathrm{qMic}=$ cociente microbiano; $\mathrm{qCO}_{2}=$ cociente metabólico; $\mathrm{qCO}_{2} \mathrm{C}_{\mathrm{org}}{ }^{-1}=$ cociente de eficiencia metabólica. ${ }^{*}$ Correlación significativa a $P<0.05 ; * *$ correlación significativa a $P<0.01 \mathrm{y} * * *$ correlación significativa a $P<0.001$. 


\section{CONCLUSIONES}

- El manejo orgánico de los cafetales afecta positivamente las propiedades químicas y microbiológicas del suelo al incrementar el carbono orgánico, el carbono hidrosoluble, la biomasa microbiana y su actividad (respiración basal) y alberga una comunidad microbiana más eficiente, en comparación con manejos tradicionales $\mathrm{y}$ convencionales.

- Los parámetros microbiológicos medidos junto con sus variables ecofisiológicas derivadas permitieron describir los diversos tipos de manejo de la zona cafetalera andina y permiten inferir sobre la mejor calidad del suelo en los sistemas de producción orgánica. La relación $\mathrm{qCO}_{2} \mathrm{C}_{\mathrm{org}}{ }^{-1}$ es sensible, fácil de determinar y enfatiza las diferencias en el contenido de carbono entre diferentes sistemas de manejo cafetaleros.

\section{AGRADECIMIENTOS}

Esta investigación fue financiada por la Oficina del Convenio Integral de Cooperación CubaVenezuela (Proyecto: "Recuperación de áreas del Caribe en proceso de desertificación. Fase I: Diagnostico de áreas vulnerables y en proceso de desertificación mediante el uso de un Sistema de Información Geográfica). El autor agradece a la editora y los revisores de Terra Latinoamericana por las observaciones para mejorar este artículo.

\section{LITERATURA CITADA}

Alef, K. 1995. Estimation of soil respiration. pp. 464-470. In: K. Alef and P. Nannipieri (eds.). Methods in applied soil microbiology and biochemistry. Academic Press, New York, NY, USA.

Almeida, E. F., R. H. P. Polizel, L. C. Gomes, F. A. S. Xavier, e E. S. Mendonca. 2007. Biomassa microbiana em sistema agroflorestais na zona da mata mineira. Rev. Bras. Agroecol. 2: 739-742.

Anderson, J. P. E. and K. H. Domsch. 1978. A physiological method for the quantitative measurement of microbial biomass in soils. Soil Biol. Biochem. 10: 215-221.doi: 10.1016/0038-0717(78)90099-8.

Anderson, T. H. 1994. Physiological analysis of microbial communities in soil: Applications and limitations. pp. 6776. In: K. Ritz, J. Dighton, and K. E. Giller (eds.). Beyond the biomass, compositional and functional analysis of soil microbial communities. Wiley-Sayce. Chichester, UK.
Anderson, T. H. 2003. Microbial eco-physiological indicators to assess soil quality. Agric. Ecosyst. Environ. 98: 285-293. doi: 10.1016/S0167-8809(03)00088-4.

Anderson, T. H. and K. M. Domsch. 1989. Ratios of microbial biomass carbon to total organic-C in arable soils. Soil Biol. Biochem. 21: 471-479. doi: 10.1016/00380717(89)90117-X.

Anderson, T. H. and K. M. Domsch. 1990. Application of ecophysiological quotients (qCO2 y qD) on microbial biomass from soil of different cropping histories. Soil Biol. Biochem. 22: 251-255. doi: 10.1016/0038-0717(90)90094-G.

Aranguren, J., G. Escalante, and R. Herrera. 1982. Nitrogen cycle of tropical perennial crops under shade trees. I. Coffee. Plant Soil 67: 247-258. doi: 10.1007/BF02182772.

Araújo, A. S. F., V. B. Santos, and R. T. R. Monteiro. 2008. Responses of soil microbial biomass and activity for practices of organic and conventional farming systems in Piauí state, Brazil. Eur. J. Soil Biol. 44: 225-230. doi: 10.1016/j.ejsobi.2007.06.001.

Arellano, R., J. Paolini, L. Vásquez y E. Mora. 2004. Producción y descomposición de la hojarasca en tres agroecosistemas de café en el estado Trujillo, Venezuela. Rev. For. Venezol. 48: 7-14.

Balota, E. L. and J. C. D. Chaves. 2011. Microbial activity in soil cultivated with different summer legumes in coffee crop. Braz. Arch. Biol. Technol. 54: 35-44. doi: 10.1590/ S1516-89132011000100005.

Beck, T., R. Öhlinger, and A. Baumgarten. 1995. Substrateinduced respiration. pp. 64-68. In: F. Schinner, R. Öhlinger, E. Kandeler, and R. Margesin (eds.). Methods in soil biology. Springer Verlag, Berlin, FRG.

Beer, J. 1987. Advantages, disadvantages and desirable characteristics of shade trees for coffee, cacao and tea. Agrofor. Syst. 5: 3-13. doi: 10.1007/BF00046410.

Beer, J., R. Muschler, D. Kass, and E. Somarriba. 1998. Shade management in coffee and cacao plantations. Agrofor. Syst. 38: 139-164. doi: 10.1023/A:1005956528316.

Berner, A., I. Hildermann, A. Fließbach, L. Pfiffner, U. Niggli, and P. Mäder. 2008. Crop yield and soil fertility response to reduced tillage under organic management. Soil Tillage Res. 101: 89-96. doi: 10.1016/j.still.2008.07.012.

Bettiol, W., R. Ghini, J. A. H. Galvão, M. A. V. Ligo, and J. L. C. Mineiro. 2002. Soil organisms in organic and conventional cropping systems. Sci. Agric. 59: 565-572. doi: 10.1590/ S0103-90162002000300023.

Böhme, L., U. Langer, and F. Böhme. 2006. Microbial biomass, enzyme activities and microbial community structure in two European long-term field experiments. Soil Biol. Biochem. 109: 141-152. doi: 10.1016/j.agee.2005.01.017.

Bosselmann, A., K. Dons, T. Oberthur, C. Smith Olsen, A. Raebild and H. Usma. 2009. The influence of shade trees on coffee quality in small holder coffee agroforestry systems in southern Colombia. Agricu. Ecosyst. Environ. 129: 253260. doi: 10.1016/j.agee.2008.09.004.

Canali, S, E. Di Bartolomeo, A. Trinchera, L. Nisini, F. Tittarelli, F. Intrigliolo, G. Roccuzzo, and M. L. Calabretta. 2009. Effect of different management strategies on soil quality of citrus orchards. Soil Use Manage. 25: 34-42. doi: 10.111/j.1475-2743.2008.00191.x. 
Cheng, W., Q. Zhang, D. C. Coleman, C. R. Carroll, and C. A. Hoffman. 1996. Is available carbon limiting microbial respiration in the rhizosphere? Soil Biol. Biochem. 28: 1283-1288. doi: 10.1016/S0038-0717(96)00138-1.

DaMatta, F. M. 2004. Ecophysiological constraints on the production of shaded and unshaded coffee: A review. Field Crops Res. 86: 90-114. doi: 10.1016/j.fcr.2003.09.001.

Dariah, A., F. Agus, and D. Maswar. 2008. Soil quality of the land under coffee-based farming system (case study at Sumberjaya, West Lampug). Ind. J. Agric. 1: 51-57.

Dilly, O. 2005. Microbial energetics in soils. pp. 123-138. In: F. Buscot and A. Varma (eds.). Microorganisms in soils: Roles in genesis and functions. Springer-Verlag. Berlin, FRG.

Dilly, O. and J. C. Munch. 1996. Microbial biomass content, basal respiration and enzyme activities during the course of decomposition of leaf litter in a black alder (Alnus glutinosa L. Gaertn) forest. Soil Biol. Biochem. 28: 1073-1081. doi: 10.1016/0038-0717(96)00075-2.

Dilly, O. and J. C. Munch. 1998. Ratios between estimates of microbial biomass content and microbial activity in soils. Biol. Fertil. Soils 27: 374-379. doi: 10.1007/ s003740050446.

Dilly, O., K. Winter, A. Lang, and J. C. Munch. 2001. Energetic eco-physiology of the soil microbiota in two landscapes of southern and northern Germany. J. Plant Nutr. Soil Sci. 164: 407-413.doi: 10.1002/1522-2624(200108)164:4<407::AIDJPLN407>3.0.CO;2-9.

Dinesh, R., S. G. Chaudhuri, A. N. Ganeshamurthy, and Ch. Dey. 2003. Changes in soil microbial indices and their relationships following deforestation and cultivation in wet tropical forests. Appl. Soil Ecol. 24: 17-26. doi: 10.1016/ S0929-1393(03)00070-2.

Escalante, E. 1985. Promising agroforestry systems in Venezuela. Agrofor. Syst. 3: 209-221. doi: 10.1007/BF00122644.

Franca, S. C., S. M. Gomez-da-Costa, and A. P. D. Silveira. 2007. Microbial activity and arbuscular mycorrhizal fungal diversity in conventional and organic citrus orchards. Biol. Agric. Hortic. 25: 91-102. doi: 10.1080/01448765.2007.9755039.

Glaeser, D. F., F. M. Mercante, M. A. M. Alves, R. F. da Silva, and O. M. Komori. 2010. Biomassa microbiana do solo sob sistemas de manejo organico em cultivos de café. Ens. Ciênc.: Ciênc. Biol. Agrár. Saúde 14: 103-114.

Gunapala, N. and K. M. Scow. 1998. Dynamics of soil microbial biomass and activity in conventional and organic farming systems. Soil Biol. Biochem. 30: 805-816. doi: 10.1016/ S0038-0717(97)00162-4.

Haggar, J., M. Barrios, M. Bolaños, M. Merlo, P. Moraga, R. Munguia, A. Ponce, S. Romero, G. Soto, C. Staver, and E. de M. F. Virginio. 2011. Coffee agroecosystem performance under full sun, shade, conventional and organic management regimes in Central America. Agrofor. Syst. 82: 285-301. doi: 10.1007/s10457-011-9392-5

Henao Jaramillo, J. 1996. El café en Venezuela. Universidad Central de Venezuela. Ediciones Biblioteca, Colección Ciencias Biológicas. Caracas, Venezuela.

Höper, H. 2006. Substrate-induced respiration. pp. 84-92. In: J. Bloem, D. W. Hopkins, and A. Benedetti (eds.). Microbiological methods for assessing soil quality. $\mathrm{CAB}$ International. Wallingford, UK.
Insam, H. and K. H. Domsch. 1988. Relationships between soil organic carbon and microbial biomass on chronosequences of reclamation sites. Microbial Ecol. 15: 177-188. doi: 10.1007/BF02011711.

Jenkinson, D. S. and J. N. Ladd. 1981. Microbial biomass in soil: Measurement and turnover. pp. 415-471. In: E. A. Paul and J. N. Ladd (eds.). Soil biochemistry, Volume 5. Marcel Dekker. New York, NY, USA.

Joergensen, R. G., P. Mäder, and A. Fließbach. 2010. Long-term effects of organic farming on fungal and bacterial residues in relation to microbial energy metabolism. Biol. Fert. Soils 46: 303-307. doi: 10.1007/s00374-009-0433-4.

Kaschuk, G., O. Alberton, and M. Hungria. 2010. Three decades of soil microbial biomass studies in Brazilian ecosystems: Lessons learned about soil quality and indications for improving sustainability. Soil Biol. Biochem. 42: 1-13. doi: 10.1016/j.soilbio.2009.08.020.

Lagomarsino, A., M. C. Moscatelli, A. Di Tizio, R. Mancinelli, S. Grego, and S. Marinari. 2009. Soil biochemical indicators as a tool to assess the short-term impact of agricultural management on changes in organic $\mathrm{C}$ in a Mediterranean environment. Ecol. Indicat. 9: 518-527. doi: 10.1016/j. ecolind.2008.07.003.

Leita, L., M. de Nobili, C. Mondini, G. Muhlbachova, L. Marchiol, G. Bragato, and M. Contin. 1999. Influence of inorganic and organic fertilization on soil microbial biomass, metabolic quotient and heavy metal bioavailability. Biol. Fertil. Soils 28: 371-376. doi: 10.1007/s003740050506.

Leite, L. F. C., F. C. Oliveira, A. Araujo, S. R. S. Galvão, J. O. Lemos, and E. Silva. 2010. Soil organic carbon and biological indicators in an acrisol under tillage systems and organic management in Northeastern Brazil. Aust. J. Soil Res. 48: 258-265. doi: 10.1071/SR09122.

Madejón, E., F. Moreno, J. M. Murillo, and F. Pelegrín. 2007. Soil biochemical response to long-term conservation tillage under semi-arid Mediterranean conditions. Soil Res. Tillage 94: 346-352. doi: 10.1016/j.still.2006.08.010.

Mäder, P., A. Fliessbach, D. Dubois, L. Gunst, P. Fried, and U. Niggli. 2002. Soil fertility and biodiversity in organic farming. Science 296: 1694-1697. doi: 10.1126/ science. 1071148.

Mamani-Pati, F., D. E. Clay, S. A. Clay, H. Smelkop, and M.A. Yujra-Callata. 2012. The influence of strata on the nutrient recycling within a tropical certified organic coffee production systems. ISRN Agronomy 2012: 1-8, ID 389280. doi: $10.5402 / 2012 / 389290$.

Marinari, S., R. Mancinelli, E. Campiglia, and S. Grego. 2006. Chemical and biological indicators of soil quality in organic and conventional farming systems in Central Italy. Ecol. Indicat. 6: 701-711. doi: 10.1016/j.ecolind.2005.08.029.

Marumoto, T., J. P. E. Anderson, and K. H. Domsch. 1982. Mineralization of nutrients from soil microbial biomass. Soil Biol. Biochem. 14: 469-475. doi: 10.1016/00380717(82)90106-7.

Melero, S., J. C. Ruiz, J. F. Herencia, and E. Madejon. 2006. Chemical and biochemical properties in a silty loam soil under conventional and organic management. Soil Tillage Res. 90: 162-170. doi: 10.1016/j.still.2005.08.016.

Moguel, P. and V. M. Toledo. 1999. Biodiversity conservation in traditional coffee systems of Mexico. Conservat. Biol. 13: 11-21. doi: 10.1046/j.1523-1739.1999.97153.x. 
Moscatelli, M. C., A. Lagomarsino, S. Marinari, P. De Angelis, and S. Grego. 2005. Soil microbial indices as bioindicators of environmental changes in a poplar plantation. Ecol. Indicat. 5: 171-179. doi: 10.1016/j.ecolind.2005.03.002.

Muschler, R. G. 2001. Shade improves coffee quality in a suboptimal coffee-zone of Costa Rica. Agrofor. Syst. 51: 131139. doi: 10.1023/A:1010603320653.

Nannipieri, P., S. Grego, and B. Ceccanti. 1990. Ecological significance of the biological activity in soil. pp. 293-355. In: J. M. Bollag and G. Stotzky (eds.) Soil Biochemistry, Volume 6. Marcel Dekker. New York, NY, USA.

Nunes, L. A. P., L. E. Dias, I. Jucksch, N. F. Barros, M. C. M. Kasuya, and M. E. F. Correia. 2009. Impacto do monocultivo de café sobre os indicadores biológicos do solo na zona da mata mineira. Ciênc. Rural 39: 2467-2474. doi: 10.1590/ S0103-84782009005000216.

Odum, E. P. 1969. The strategy of ecosystem development. Science 164: 262-270. doi: 10.1126/science.164.3877.262.

Okur, N., H. H. Kayikcioglu, F. Ates, and B. Yagmur. 2015. A comparison of soil quality and yield parameters under organic and conventional vineyard systems in Mediterranean conditions (west Turkey). Biol. Agric. Hortic. 32: 1-12. doi: 10.1080/01448765.2015.1033645.

Pabst, H., A. Kuhnel, and Y. Kuzyakov. 2013. Effect of land-use and elevation on microbial biomass and water extractable carbon in soils of Mt. Kilimanjaro ecosystems. Appl. Soil Ecol. 67: 10-19. doi: 10.1016/j.apsol.2013.02.006.

Partelli, F. L., H. D. Vieira, E. P. de Brito Ferreira, A. P. Viana, M. A. Martins, and S. Urquiaga. 2012. Chemical and microbiological soil characteristics under conventional and organic coffee production systems. Commun. Soil Sci. Plant Anal. 43: 847-864. doi: 10.1080/00103624.2012.648470.

Payán, F., D. L. Jones, J. Beer, and J. M. Harmand. 2009. Soil characteristics below Erythrina poeppigiana in organic and conventional Costa Rican coffee plantations. Agrofor. Syst. 76: 81-93. doi: 10.1007/s10457-008-9201-y.

Pell, M., J. Stenström, and U. Granhall. 2006. Soil respiration. pp. 117-126. In: J. Bloem, D. W. Hopking, and A. Benedetti (eds.). Microbiological methods for assessing soil quality. CAB International. Wallingford, UK.

Perfecto, I. and I. Armbrecht. 2003. The coffee agroecosystem in the neotropics: Combining ecological and economic goals. pp. 159-194. In: J. Vandermeer (ed.). Tropical agroecosystems (Advances in agroecology). CRC Press. Boca Raton, FL, USA.

Pimentel, M. S., A. M. de Aquino, M. E. Fernandes Correia, J. Ribeiro Costa, M. S. Freiri Ricci y H. De-Polli. 2006. Atributos biológicos do solo sob manejo orgánico de caffeiro, pastagem e floresta em Região de Medio Paraiba Fluminense-RJ. Coffee Sci. (Lavras) 1: 85-93. doi: 10.1590/ S0100-204X2011000500013.

Quintero, J. S. y M. Ataroff. 1998. Contenido y flujo de nitrógeno en la biomasa y hojarasca de un cafetal a plena exposición solar en Los Andes venezolanos. Rev. Fac. Agron. (LUZ) 15: 501-514.

Rapidel, B., C. Allinne, C. Cerdán, L. Meylan, E. V. Filho y J. Avelino. 2015. Efectos ecológicos y productivos del asocio de árboles de sombra con café en sistemas agroforestales. pp. 5-19. In: F. Montagnini, E. Somarriba, E. Murgueitio, M. Fassola y B. Eibl (eds.). Sistemas agroforestales: Funciones productivas, socioeconómicas y ambientales. Serie técnica. Informe técnico 402. CATIE, Turrialba, Costa Rica. CIPAV, Cali, Colombia. ISBN: 978-958-9386-74-3

Saffigna, P. G., D. S. Powlson, P. C. Brookes, and G. A. Thomas. 1989. Influence of sorghum residues and tillage on soil organic matter and soil microbial biomass in an Australian vertisol. Soil Biol. Biochem. 21: 759-765. doi: 10.1016/0038-0717(89)90167-3.

Sánchez de León, Y., E. de Melo, G. Soto, J. Johnson-Maynard, and J. Lugo-Pérez. 2006. Earthworm populations, microbial biomass and coffee production in different experimental agroforestry management systems in Costa Rica. Caribbean J. Sci. 42: 397-409. doi: 10.18475/cjos.v42i3.a.

Santos, V. D., A. S. F. Araújo, L. F. C. Leite, L. A. P. L. Nunes, and W. J. Melo. 2012. Soil microbial biomass and organic matter fractions during transition from conventional to organic farming systems. Geoderma 170: 227-231. doi: 10.1016/j.geoderma.2011.11.007.

Severino, L. S. y T. S. de Oliveira. 1999. Sistema de cultivo sombreado do cafeeiro (Coffea arabica L.) na região de Baturite, Ceará. Rev. Ceres 46: 635-652.

Sims, J. R. and V. A. Haby. 1971. Simplified colorimetric determination of soil organic matter. Soil Sci. 112: 137-141. doi: 10.1097/00010694-197108000-00007.

Souza, H. N., R. G. M. de Goede, L. Brussaard, I. M. Cardoso, E. M. G. Duarte, R. B. A. Fernandes, L. C. Gomes, and M. M. Pulleman. 2012. Protective shade, tree diversity and soil properties in coffee agroforestry systems in the Atlantic rainforest biome. Agric. Ecosyst. Environ. 146: 179-196. doi: 10.1016/j.agee.2011.11.007.

Sparling, G. P. 1992. Ratio of microbial biomass carbon to soil organic carbon as a sensitive indicator of changes in soil organic matter. Aust. J. Soil Res. 30: 195-207. doi: 10.1071/ SR9920195.

Theodoro, V. C., M. I. N. Alvarenga, R. J. Guimarães e M. Mourão Júnior. 2003. Carbono da biomassa microbiana e micorriza em solo sob mata nativa e agroecosistemas cafeeiros. Acta Scient. Agron. (Maringá) 25: 147-153.

Tu, C., F. J. Louws, N. G. Creamer, J. P. Mueller, C. Brownie, K. Fager, M. Bell, and S. Hu. 2006. Responses of soil microbial biomass and $\mathrm{N}$ availability to transition strategies from conventional o organic farming systems. Agric. Ecosyst. Environ. 113: 206-215. doi: 10.1016/j.agee.2005.09.013.

Van Gestel, M., J. N. Ladd, and M. Amato. 1992. Microbial biomass responses to seasonal change and imposed drying regimes at increasing depths of undisturbed topsoil profiles. Soil Biol. Biochem. 24: 103-111. doi: 10.1016/00380717(92)90265-y.

Velmourougane, K. 2016. Impact of organic and conventional systems of coffee farming on soil properties and culturable microbial diversity. Scientifica 2016: 1-9. ID 3604026. doi: 10.1155/2016/3604026.

Wardle, D. A. and A. Ghani. 1995. A critique of the microbial metabolic quotient $(\mathrm{qCO} 2)$ as bioindicator of disturbance and ecosystem development. Soil Biol. Biochem. 27: 16011610. doi: 10.1016/0038-0717(95)00093-T.

Willer, H. and J. Lernoud. 2016. The World of organic agriculture. Statistics and Emerging Trends 2016. Research Institute of Organic Agriculture (FiBL), Frick, and IFOAM-Organic International, Bonn. ISBN: 978-3-03736-307-2. 